\title{
Quantum local-field corrections and spontaneous decay
}

\author{
Stefan Scheel, Ludwig Knöll, Dirk-Gunnar Welsch \\ Theoretisch-Physikalisches Institut, Friedrich-Schiller-Universität Jena, Max-Wien-Platz 1, 07743 Jena, Germany \\ Stephen M. Barnett \\ Department of Physics and Applied Physics, University of Strathclyde, Glasgow G4 ONG, Scotland
}

(June 28, 2006)

A recently developed scheme [S. Scheel, L. Knöll, and D.G. Welsch, Phys. Rev. A 58, 700 (1998)] for quantizing the macroscopic electromagnetic field in linear dispersive and absorbing dielectrics satisfying the Kramers-Kronig relations is used to derive the quantum local-field correction for the standard virtual-sphere-cavity model. The electric and magnetic local-field operators are shown to be consistent with QED only if the polarization noise is fully taken into account. It is shown that the polarization fluctuations in the local field can dramatically change the spontaneous decay rate, compared with the familiar result obtained from the classical local-field correction. In particular, the spontaneous emission rate strongly depends on the radius of the local-field virtual cavity.

42.50.-p,42.50.Ct,42.50.Lc

\section{INTRODUCTION}

Spontaneous emission by an excited atom is one of the most studied examples of a quantum process and may be attributed, at least in part, to fluctuations in the electromagnetic vacuum [1]. The vacuum field is modified by the local environment and this, in turn, leads to a modification of the spontaneous emission rate. In this way the spontaneous emission rate can be changed by embedding the radiating atom inside a dielectric host [2 7], or by changing the boundary conditions either by a cavity 8 12] or a suitable surface [13,14]. Recent experiments have examined the emission by atoms embedded in dielectric hosts [15 17] and have encouraged us to reexamine the problem of local-field corrections to the bulk modification of the spontaneous decay rate.

The total decay rate $\Gamma$ can be split into two parts,

$$
\Gamma=\Gamma^{\perp}+\Gamma^{\|}
$$

in which we associate the transverse decay rate $\Gamma^{\perp}$ and the longitudinal decay rate $\Gamma^{\|}$with the contributions of the transverse and longitudinal fields respectively. The dielectric-induced modification of the spontaneous emission rate in free space can be ascribed to two effects associated with the bulk (macroscopic) field in the medium and the other arising from the local (microscopic) field. The bulk field correction multiplies the rate by the refractive index at the transition frequency [3, 4]. Localfield corrections present more of a problem and have a form that is strongly model-dependent. For the ClausiusMosotti model, which introduces a virtual cavity surrounding the atom, a classical treatment of the local-field corrections leads to the form [3, 4 ]

$$
\Gamma_{\mathrm{cl}}^{\perp}=\eta\left(\omega_{A}\right)\left|\frac{\epsilon\left(\omega_{A}\right)+2}{3}\right|^{2} \Gamma_{0}
$$

for the transverse decay rate of an atom in a bulk dielectric of refractive index $n(\omega)=\sqrt{\epsilon(\omega)}=\eta(\omega)+i \kappa(\omega)$. In Eq. (2), $\Gamma_{0}=\omega_{A}^{3} \mu^{2} /\left(3 \pi c^{3} \hbar \epsilon_{0}\right)$ is the free-space spontaneous emission rate, where $\omega_{A}$ and $\mu$ are, respectively, the atomic transition frequency and the dipole transition matrix element. The local field correction in Eq. (2) arises from writing the local electric field in terms of the macroscopic electric field and the commonly used induced polarization field. It does not, however, take account of the fluctuating component of polarization associated with absorption losses. In this paper we investigate the changes that arise within the Clausius-Mosotti model when this fluctuating component is included.

Recently, a scheme for quantizing the electromagnetic field in an arbitrary linear dielectric medium has been proven to be consistent with QED 18. It relies on the introduction of an appropriately chosen infinite set of basic-field operators 19 21 and their connection to the electromagnetic field operators via the classical Green function. This scheme is a generalization of the approach introduced by Huttner and Barnett 22] based on a Hopfield model 23. of a homogeneous dielectric using Fano diagonalization 24 to obtain collective (polariton) excitations of the electromagnetic field, the polarization and the reservoir. As we will show below, the scheme can also be applied to local-field quantization that is consistent with QED and used for calculating the modified spontaneous decay rate including the local quantum effects of the fluctuating medium polarization.

The paper is organized as follows. After a short review of the quantization scheme in Sect. III we introduce the quantum local-field correction in Sect. [II]. We then apply the scheme to the calculation of the spontaneous decay rates in Sect. IV followed by some concluding remarks in Sect. V. Details of the calculation will be given in the Appendix. 


\section{QUANTIZATION SCHEME}

We begin with a brief review of the quantization scheme used throughout the paper. Further details can be found in $[18$ 20]. The spectral decomposition of the electric and magnetic field operators is given by

$$
\begin{aligned}
& \hat{\mathbf{E}}(\mathbf{r})=\int_{0}^{\infty} d \omega \underline{\hat{\mathbf{E}}}(\mathbf{r}, \omega)+\text { H.c. }, \\
& \hat{\mathbf{B}}(\mathbf{r})=\int_{0}^{\infty} d \omega \underline{\hat{\mathbf{B}}}(\mathbf{r}, \omega)+\text { H.c. },
\end{aligned}
$$

where $\underline{\hat{\mathbf{E}}}(\mathbf{r}, \omega)$ and $\underline{\hat{\mathbf{B}}}(\mathbf{r}, \omega)$ satisfy Maxwell's equations

$$
\begin{gathered}
\nabla \cdot \underline{\hat{\mathbf{B}}}(\mathbf{r}, \omega)=0, \\
\nabla \cdot\left[\epsilon_{0} \epsilon(\mathbf{r}, \omega) \underline{\hat{\mathbf{E}}}(\mathbf{r}, \omega)\right]=\underline{\hat{\rho}}(\mathbf{r}, \omega), \\
\nabla \times \underline{\hat{\mathbf{E}}}(\mathbf{r}, \omega)=i \omega \underline{\hat{\mathbf{B}}}(\mathbf{r}, \omega), \\
\nabla \times \underline{\hat{\mathbf{B}}}(\mathbf{r}, \omega)=-i \frac{\omega}{c^{2}} \epsilon(\mathbf{r}, \omega) \underline{\hat{\mathbf{E}}}(\mathbf{r}, \omega)+\mu_{0} \hat{\hat{j}}(\mathbf{r}, \omega)
\end{gathered}
$$

$\left[\epsilon(\mathbf{r}, \omega)=\epsilon_{R}(\mathbf{r}, \omega)+i \epsilon_{I}(\mathbf{r}, \omega)\right.$ is the permittivity]. The operator noise current density $\hat{\mathbf{j}}(\mathbf{r}, \omega)$ and the operator noise charge density $\hat{\rho}(\mathbf{r}, \omega)$, which had to be introduced in order to be consistent with the dissipation-fluctuation theorem, are related to the noise polarization $\underline{\hat{\mathbf{P}}}^{N}(\mathbf{r}, \omega)$ as

$$
\begin{aligned}
& \underline{\hat{\mathbf{j}}}(\mathbf{r}, \omega)=-i \omega \underline{\hat{\mathbf{P}}}^{N}(\mathbf{r}, \omega), \\
& \underline{\hat{\rho}}(\mathbf{r}, \omega)=-\nabla \cdot \underline{\hat{\mathbf{P}}}^{N}(\mathbf{r}, \omega)
\end{aligned}
$$

and satisfy the equation of continuity

$$
\nabla \cdot \underline{\hat{\mathbf{j}}}(\mathbf{r}, \omega)=i \omega \underline{\hat{\rho}}(\mathbf{r}, \omega) .
$$

The operator noise current density $\underline{\hat{\mathbf{j}}}(\mathbf{r}, \omega)$ is obtained from a bosonic vector field $\hat{\mathbf{f}}(\mathbf{r}, \omega)$,

$$
\begin{aligned}
\hat{\mathbf{j}}(\mathbf{r}, \omega) & =\omega \sqrt{\frac{\hbar \epsilon_{0}}{\pi} \epsilon_{I}(\mathbf{r}, \omega)} \hat{\mathbf{f}}(\mathbf{r}, \omega), \\
{\left[\hat{f}_{i}(\mathbf{r}, \omega), \hat{f}_{j}^{\dagger}\left(\mathbf{r}^{\prime}, \omega^{\prime}\right)\right] } & =\delta_{i j} \delta\left(\mathbf{r}-\mathbf{r}^{\prime}\right) \delta\left(\omega-\omega^{\prime}\right), \\
{\left[\hat{f}_{i}(\mathbf{r}, \omega), \hat{f}_{j}\left(\mathbf{r}^{\prime}, \omega^{\prime}\right)\right] } & =\left[\hat{f}_{i}^{\dagger}(\mathbf{r}, \omega), \hat{f}_{j}^{\dagger}\left(\mathbf{r}^{\prime}, \omega^{\prime}\right)\right]=0 .
\end{aligned}
$$

The quantization scheme implies that all electromagnetic field operators can be expressed in terms of the basic fields $\hat{\mathbf{f}}(\mathbf{r}, \omega)$, which may be regarded as being the collective excitations of the electromagnetic field, the medium polarization, and the reservoir. For example, the electric-field operator $\underline{\hat{\mathbf{E}}}(\mathbf{r}, \omega)$ satisfies the partial differential equation

$$
\nabla \times \nabla \times \underline{\hat{\mathbf{E}}}(\mathbf{r}, \omega)-\frac{\omega^{2}}{c^{2}} \epsilon(\mathbf{r}, \omega) \underline{\hat{\mathbf{E}}}(\mathbf{r}, \omega)=i \mu_{0} \omega \underline{\hat{\mathbf{j}}}(\mathbf{r}, \omega),
$$

such that

$$
\underline{\hat{E}}_{i}(\mathbf{r}, \omega)=i \mu_{0} \int d^{3} \mathbf{s} \omega G_{i j}(\mathbf{r}, \mathbf{s}, \omega) \hat{j}_{j}(\mathbf{s}, \omega),
$$

where $G_{i j}(\mathbf{r}, \mathbf{s}, \omega)$ is the tensor-valued Green function of the classical problem. It can then be proven 118 that this quantization scheme is fully consistent with QED for arbitrary linear dielectrics.

\section{QUANTUM LOCAL-FIELD CORRECTION}

If we think of an atom located at some space point $\mathbf{r}_{A}$ inside the dielectric, then the macroscopic field of Sect. II will not, in fact, be the field felt by the atom. From classical electrodynamics we know that we should introduce what is called the local field at the location of the atom [25. There are essentially two ways of introducing the local field. First, one could cut out a real cavity [2] (most commonly a sphere) around the atom and calculate, in our scheme, the electric field inside the cavity according to Eq. (16). This would lead us to introduce the electric field operator $\underline{\hat{\mathbf{E}}}^{\mathrm{loc}}(\mathbf{r}, \omega)$ by the relation

$$
\underline{\hat{E}}_{i}^{\mathrm{loc}}(\mathbf{r}, \omega)=i \mu_{0} \int d^{3} \mathbf{s} \omega G_{i j}^{\mathrm{inh}}(\mathbf{r}, \mathbf{s}, \omega) \underline{j}_{j}(\mathbf{s}, \omega),
$$

where $G_{i j}^{\mathrm{inh}}(\mathbf{r}, \mathbf{s}, \omega)$ is the Green function of the classical problem of an inhomogeneous medium that consists of the real cavity surrounded by the dielectric in which the atom is embedded.

To avoid the solution of the inhomogeneous problem, commonly a simpler virtual-cavity model of ClausiusMosotti-type is used. In this model the local field is (approximately) related to the macroscopic field, which in our scheme can be obtained according Eq. (16), with the Green function for the bulk-medium problem. In classical optics the electric field is usually corrected by adding a term proportional to the polarization,

$$
\underline{\mathbf{E}}^{\mathrm{loc}}(\mathbf{r}, \omega)=\underline{\mathbf{E}}(\mathbf{r}, \omega)+\frac{1}{3 \epsilon_{0}} \underline{\mathbf{P}}(\mathbf{r}, \omega),
$$

where

$$
\underline{\mathbf{P}}(\mathbf{r}, \omega)=\epsilon_{0}[\epsilon(\mathbf{r}, \omega)-1] \underline{\mathbf{E}}(\mathbf{r}, \omega),
$$

from which it follows that

$$
\underline{\mathbf{E}}^{\mathrm{loc}}(\mathbf{r}, \omega)=\frac{1}{3}[\epsilon(\mathbf{r}, \omega)+2] \underline{\mathbf{E}}(\mathbf{r}, \omega) .
$$

This classically corrected local field is just the field used for the derivation of the rate formula (2).

In classical optics Eq. (19) corresponds to the zerotemperature limit. It does not, however, hold in operator form in quantum optics. Regarding Eq. 19) as an operator equation would strongly contradict the QED equal-time commutation relations in general, so that the operator counterpart of Eq. (20) will not be a true electric field. In order to obtain a canonical operator equation, 
we have to complement Eq. (19) by a noise polarization $\underline{\hat{\mathbf{P}}}^{N}(\mathbf{r}, \omega)$ :

$$
\underline{\hat{\mathbf{P}}}(\mathbf{r}, \omega)=\epsilon_{0}[\epsilon(\mathbf{r}, \omega)-1] \underline{\hat{\mathbf{E}}}(\mathbf{r}, \omega)+\underline{\hat{\mathbf{P}}}^{N}(\mathbf{r}, \omega) .
$$

Using the quantization scheme in Sec. II, from Eq. (9) the noise polarization $\underline{\hat{\mathbf{P}}}^{N}(\mathbf{r}, \omega)$ is given by

$$
\underline{\hat{\mathbf{P}}}^{N}(\mathbf{r}, \omega)=-\frac{1}{i \omega} \hat{\mathbf{j}}(\mathbf{r}, \omega)=i \sqrt{\frac{\hbar \epsilon_{0}}{\pi} \epsilon_{I}(\mathbf{r}, \omega)} \hat{\mathbf{f}}(\mathbf{r}, \omega) .
$$

Combining Eqs. (18) and (21), a local electric-field operator

$$
\underline{\hat{\mathbf{E}}}^{\mathrm{loc}}(\mathbf{r}, \omega)=\frac{1}{3}[\epsilon(\mathbf{r}, \omega)+2] \underline{\hat{\mathbf{E}}}(\mathbf{r}, \omega)+\frac{1}{3 \epsilon_{0}} \underline{\hat{\mathbf{P}}}^{N}(\mathbf{r}, \omega)
$$

can be introduced, with $\underline{\hat{\mathbf{P}}}^{N}(\mathbf{r}, \omega)$ being given in Eq. (22).

In order to prove the consistency of the quantized local field with QED, we compute the (equal-time) commutation relation between the fundamental local fields $\hat{\mathbf{E}}^{\text {loc }}(\mathbf{r})$ and $\hat{\mathbf{B}}^{\text {loc }}(\mathbf{r})$. For this purpose we note that the local magnetic-field operator is given by, on using Eqs. (7),

$$
\underline{\hat{\mathbf{B}}}^{\mathrm{loc}}(\mathbf{r}, \omega)=\nabla \times \mathcal{P} \frac{1}{i \omega} \underline{\hat{\mathbf{E}}}^{\mathrm{loc}}(\mathbf{r}, \omega),
$$

where the symbol $\mathcal{P}$ stands for the principal part. Recalling Eqs. (3) and (4), the local-field operators in real space are

$$
\hat{\mathbf{E}}^{\mathrm{loc}}(\mathbf{r})=\int_{0}^{\infty} d \omega \underline{\hat{\mathbf{E}}}^{\mathrm{loc}}(\mathbf{r}, \omega)+\text { H.c. }
$$

and

$$
\hat{\mathbf{B}}^{\mathrm{loc}}(\mathbf{r})=\int_{0}^{\infty} d \omega \underline{\hat{\mathbf{B}}}^{\mathrm{loc}}(\mathbf{r}, \omega)+\text { H.c.. }
$$

Expressing the local electric and magnetic fields in terms of the basic fields $\hat{\mathbf{f}}(\mathbf{r}, \omega)$, from the calculation given in App. A it is found that

$$
\begin{aligned}
& {\left[\hat{E}_{i}^{\mathrm{loc}}(\mathbf{r}), \hat{B}_{k}^{\mathrm{loc}}\left(\mathbf{r}^{\prime}\right)\right]} \\
& =-\frac{i \hbar}{\epsilon_{0}} \epsilon_{i k l} \partial_{l}^{r} \delta\left(\mathbf{r}-\mathbf{r}^{\prime}\right)\left\{1+\frac{1}{9}[\epsilon(\mathbf{r}, 0)-1]\right\},
\end{aligned}
$$

and it is easily seen that

$$
\left[\hat{E}_{i}^{\text {loc }}(\mathbf{r}), \hat{E}_{k}^{\text {loc }}\left(\mathbf{r}^{\prime}\right)\right]=\left[\hat{B}_{i}^{\text {loc }}(\mathbf{r}), \hat{B}_{k}^{\text {loc }}\left(\mathbf{r}^{\prime}\right)\right]=0 .
$$

The result reveals that the local-electric field operator (23) and the associated magnetic field operator (24) can be regarded as being consistent with quantum theory, provided that the (real) static permittivity $\epsilon_{S}(\mathbf{r})=\epsilon(\mathbf{r}, 0)$ satisfies the condition

$$
\frac{\epsilon_{S}(\mathbf{r})}{10} \ll 1
$$

Equivalently, the static refractive index $n_{S}(\mathbf{r})=\sqrt{\epsilon_{S}(\mathbf{r})}$ must be small compared with $\sqrt{10} \approx 3.16$.

It should be noted that a term proportional to the $\delta$ function $\delta(\omega)$ can be added to the right-hand side of Eq. (24) in order to recover Ampere's law when the equation is multiplied by $\omega$. Obviously, this ambiguity reflects the fact that the static magnetic field cannot be inferred from the static electric field. From a simple calculation it can be shown that such a term does not change the commutation relation (27). Since it is only relevant at zero frequency, it does not play any role in the calculation of the decay at transition frequency $\omega_{A}$.

In order to take into account a possible deviation of the symmetry of the material from cubic symmetry, a structure constant $s$ can be included in Eq. (18) such that 26]

$$
\underline{\mathbf{E}}^{\mathrm{loc}}(\mathbf{r}, \omega)=\underline{\mathbf{E}}(\mathbf{r}, \omega)+\frac{1}{\epsilon_{0}}\left[\frac{1}{3}+s\right] \underline{\mathbf{P}}(\mathbf{r}, \omega) .
$$

Regarding this equation as an operator equation with $\hat{\mathbf{P}}(\mathbf{r}, \omega)$ from Eq. (21) and following the line in Appendix A, it can be seen that Eq. (27) changes to

$$
\begin{aligned}
& {\left[\hat{E}_{i}^{\text {loc }}(\mathbf{r}), \hat{B}_{k}^{\text {loc }}\left(\mathbf{r}^{\prime}\right)\right]} \\
& =-\frac{i \hbar}{\epsilon_{0}} \epsilon_{i k l} \partial_{l}^{r} \delta\left(\mathbf{r}-\mathbf{r}^{\prime}\right)\left\{1+\frac{\alpha^{2}}{9}[\epsilon(\mathbf{r}, 0)-1]\right\},
\end{aligned}
$$

where the parameter $\alpha$ in related to $s$ by

$$
\alpha=1+3 s .
$$

Thus, consistency with quantum theory is achieved, if the condition

$$
\epsilon_{S}(\mathbf{r}) \ll 9 \alpha^{-2}+1
$$

is fulfilled.

\section{SPONTANEOUS DECAY RATE}

The spontaneous decay rate of a (two-level) atom at transition frequency $\omega_{A}$ placed at point $\mathbf{r}_{A}$ is given by

$$
\Gamma=\frac{2 \pi}{\hbar^{2}} \int d \omega \mu_{i}\left\langle 0\left|\underline{\hat{E}}_{i}^{\text {loc }}(\mathbf{r}, \omega) \underline{\hat{E}}_{j}^{\text {loc } \dagger}\left(\mathbf{r}_{A}, \omega_{A}\right)\right| 0\right\rangle \mu_{j}
$$

$\left(\mathbf{r} \rightarrow \mathbf{r}_{A}\right)$. In what follows we consider a homogeneous bulk material, i.e., $\epsilon(\mathbf{r}, \omega) \equiv \epsilon(\omega)$, and assume that the inequality (29) is fulfilled. Using Eq. (23), the vacuum expectation value of the local electric-field operators in the limit $\mathbf{r} \rightarrow \mathbf{r}_{A}$ can be written as the sum

$$
\begin{aligned}
& \left\langle 0\left|\underline{\underline{E}}_{i}^{\mathrm{loc}}(\mathbf{r}, \omega) \underline{\hat{E}}_{j}^{\text {loc } \dagger}\left(\mathbf{r}_{A}, \omega^{\prime}\right)\right| 0\right\rangle \\
& =\frac{\epsilon(\omega)+2}{3} \frac{\epsilon^{*}\left(\omega^{\prime}\right)+2}{3}\left\langle 0\left|\underline{\hat{E}}_{i}(\mathbf{r}, \omega) \underline{\hat{E}}_{j}^{\dagger}\left(\mathbf{r}_{A}, \omega^{\prime}\right)\right| 0\right\rangle \\
& \quad+\frac{1}{9 \epsilon_{0}^{2}}\left\langle 0\left|\underline{\hat{P}}_{i}^{N}(\mathbf{r}, \omega) \underline{\hat{P}}_{j}^{N \dagger}\left(\mathbf{r}_{A}, \omega^{\prime}\right)\right| 0\right\rangle
\end{aligned}
$$




$$
\begin{aligned}
& +\frac{\epsilon(\omega)+2}{9 \epsilon_{0}}\left\langle 0\left|\underline{\hat{E}}_{i}(\mathbf{r}, \omega) \underline{\hat{P}}_{j}^{N \dagger}\left(\mathbf{r}_{A}, \omega^{\prime}\right)\right| 0\right\rangle \\
& +\frac{\epsilon^{*}\left(\omega^{\prime}\right)+2}{9 \epsilon_{0}}\left\langle 0\left|\underline{\hat{P}}_{i}^{N}(\mathbf{r}, \omega) \underline{\hat{E}}_{j}^{\dagger}\left(\mathbf{r}_{A}, \omega^{\prime}\right)\right| 0\right\rangle,
\end{aligned}
$$

where $\hat{\mathbf{E}}(\mathbf{r}, \omega)$ and $\underline{\hat{\mathbf{P}}}^{N}(\mathbf{r}, \omega)$ are given by Eqs. (16) and (22) respectively. The Green function $G_{i j}\left(\mathbf{r}, \mathbf{r}_{A}, \omega_{A}\right)$ for the bulk material in the limit $\mathbf{r} \rightarrow \mathbf{r}_{A}$ has the form

$$
G_{i j}\left(\mathbf{R}, \omega_{A}\right)=G_{i j}^{\perp}\left(\mathbf{R}, \omega_{A}\right)+G_{i j}^{\|}\left(\mathbf{R}, \omega_{A}\right)
$$

$\left(\mathbf{R}=\mathbf{r}-\mathbf{r}_{A}\right)$, where

$$
\begin{aligned}
G_{i j}^{\perp}\left(\mathbf{R}, \omega_{A}\right) & =\frac{1}{4 \pi}\left\{\frac{R_{i} R_{j}}{2 R^{3}}+\frac{\delta_{i j}}{2 R}\right. \\
+\frac{2 i \omega_{A}}{3 c} & {\left.\left[\eta\left(\omega_{A}\right)+i \kappa\left(\omega_{A}\right)\right] \delta_{i j}\right\}+\mathcal{O}(R) }
\end{aligned}
$$

and

$$
\begin{aligned}
& G_{i j}^{\|}\left(\mathbf{R}, \omega_{A}\right)=-\frac{c^{2}}{4 \pi \omega_{A}^{2} \epsilon\left(\omega_{A}\right)} \\
& \quad \times\left[\frac{4 \pi}{3} \delta_{i j} \delta(\mathbf{R})+\left(\delta_{i j}-\frac{3 R_{i} R_{j}}{R^{2}}\right) \frac{1}{R^{3}}\right]+\mathcal{O}(R)
\end{aligned}
$$

are the transverse and longitudinal parts respectively. We see that the real part of the transverse Green function as well as the longitudinal part itself diverge as $R \rightarrow 0$, reflecting the fact that a macroscopic approach is valid only to some appropriately fixed scale $\bar{R}$ which exceeds the average distance of two atoms in the dielectric. Following [4], we average the divergent terms in the decay rate over a sphere of radius $\bar{R}$. We will see later that one can estimate an upper bound for $\bar{R}$ from the decay rate itself.

The first term on the right-hand side in Eq. (35) gives the contribution to the decay rate with the classically corrected local field [3, 4 ,

$$
\begin{aligned}
& \Gamma_{\mathrm{cl}}=\Gamma_{\mathrm{cl}}^{\perp}+\Gamma_{\mathrm{cl}}^{\|} \\
& =\Gamma_{0}\left|\frac{\epsilon\left(\omega_{A}\right)+2}{3}\right|^{2}\left[\eta\left(\omega_{A}\right)+\frac{3 \epsilon_{I}\left(\omega_{A}\right)}{2\left|\epsilon\left(\omega_{A}\right)\right|^{2}}\left(\frac{c}{\omega_{A} \bar{R}}\right)^{3}\right],
\end{aligned}
$$

with the transverse rate $\Gamma_{\mathrm{cl}}^{\perp}$ being given in Eq. (2). The second term in Eq. (35) is purely a contribution of the noise polarization field and is given by

$$
\begin{aligned}
\frac{1}{9 \epsilon_{0}^{2}}\left\langle 0\left|\underline{\hat{P}}_{i}^{N}(\mathbf{r}, \omega) \underline{\hat{P}}_{j}^{N \dagger}\left(\mathbf{r}_{A}, \omega^{\prime}\right)\right| 0\right\rangle \\
\quad=\frac{\hbar \epsilon_{I}(\omega)}{9 \pi \epsilon_{0}} \delta_{i j} \delta(\mathbf{R}) \delta\left(\omega-\omega^{\prime}\right) .
\end{aligned}
$$

The cross-terms mixing the macroscopic electric field and the noise polarization field give rise to the contribution

$$
\begin{aligned}
& \frac{\epsilon(\omega)+2}{9 \epsilon_{0}}\left\langle 0\left|\hat{E}_{i}(\mathbf{r}, \omega) \underline{\hat{P}}_{j}^{N \dagger}\left(\mathbf{r}_{A}, \omega^{\prime}\right)\right| 0\right\rangle \\
& \quad+\frac{\epsilon^{*}\left(\omega^{\prime}\right)+2}{9 \epsilon_{0}}\left\langle 0\left|\underline{\hat{P}}_{i}^{N}(\mathbf{r}, \omega) \underline{\hat{E}}_{j}^{\dagger}\left(\mathbf{r}_{A}, \omega^{\prime}\right)\right| 0\right\rangle \\
& =\frac{2 \omega^{2} \hbar}{3 \pi c^{2} \epsilon_{0}} \epsilon_{I}(\omega) \operatorname{Re}\left[\frac{\epsilon(\omega)+2}{3} G_{i j}(\mathbf{R}, \omega)\right] \delta\left(\omega-\omega^{\prime}\right) .
\end{aligned}
$$

Hence, the total decay rate reads

$$
\begin{aligned}
\Gamma & =\Gamma_{\mathrm{cl}}+\frac{2}{9 \hbar \epsilon_{0}} \epsilon_{I}\left(\omega_{A}\right) \mu_{i} \mu_{j} \overline{\delta_{i j} \delta(\mathbf{R})} \\
& +\frac{4}{3 \hbar \epsilon_{0}} \frac{\omega_{A}^{2}}{c^{2}} \epsilon_{I}\left(\omega_{A}\right) \mu_{i} \mu_{j} \operatorname{Re}\left[\frac{\epsilon\left(\omega_{A}\right)+2}{3} \overline{G_{i j}\left(\mathbf{R}, \omega_{A}\right)}\right] .
\end{aligned}
$$

Equation (42) is remarkable in the sense that inclusion of the polarization noise in the local field gives rise to a term that only results from that noise and leads to a dependence of the decay rate on the real part of the Green function. We average the $\delta$ tensor

$$
\delta_{i j} \delta(\mathbf{R})=\delta_{i j}^{\perp}(\mathbf{R})+\delta_{i j}^{\|}(\mathbf{R})
$$

and the Green tensor (36) over the sphere and obtain

$$
\begin{gathered}
\overline{\delta_{i j}^{\perp}(\mathbf{R})}=2 \overline{\delta_{i j}^{\|}(\mathbf{R})}=\frac{1}{2 \pi \bar{R}^{3}} \delta_{i j}, \\
\operatorname{Re} \overline{G_{i j}^{\perp}\left(\mathbf{R}, \omega_{A}\right)}=\left[\frac{1}{4 \pi \bar{R}}-\frac{\omega_{A} \kappa\left(\omega_{A}\right)}{6 \pi c}\right] \delta_{i j}, \\
\operatorname{Re} \overline{G_{i j}^{\|}\left(\mathbf{R}, \omega_{A}\right)}=-\frac{c^{2} \epsilon_{R}\left(\omega_{A}\right)}{4 \pi \omega_{A}^{2}\left|\epsilon\left(\omega_{A}\right)\right|^{2} \bar{R}^{3}} \delta_{i j}, \\
\operatorname{Im} \overline{G_{i j}^{\perp}\left(\mathbf{R}, \omega_{A}\right)}=\frac{\omega_{A} \eta\left(\omega_{A}\right)}{6 \pi c} \delta_{i j}, \\
\operatorname{Im} \overline{G_{i j}^{\|}\left(\mathbf{R}, \omega_{A}\right)}=\frac{c^{2} \epsilon_{I}\left(\omega_{A}\right)}{4 \pi \omega_{A}^{2}\left|\epsilon\left(\omega_{A}\right)\right|^{2} \bar{R}^{3}} \delta_{i j},
\end{gathered}
$$

and Eq. (42) can be given in the form of Eq. (1), where $\Gamma^{\perp}$ and $\Gamma^{\|}$read as

$$
\begin{aligned}
\Gamma^{\perp}= & \Gamma_{0}\left\{\eta\left(\omega_{A}\right)\left[\left|\frac{\epsilon\left(\omega_{A}\right)+2}{3}\right|^{2}-\frac{2 \epsilon_{I}^{2}\left(\omega_{A}\right)}{9}\right]\right. \\
& -\epsilon_{I}\left(\omega_{A}\right)\left[\epsilon_{R}\left(\omega_{A}\right)+2\right]\left[\frac{2}{9} \kappa\left(\omega_{A}\right)-\frac{c}{3 \omega_{A} \bar{R}}\right] \\
& \left.+\frac{c^{3} \epsilon_{I}\left(\omega_{A}\right)}{3 \omega_{A}^{3} \bar{R}^{3}}\right\}
\end{aligned}
$$

and

$$
\begin{aligned}
\Gamma^{\|}= & \frac{\mu^{2} \epsilon_{I}\left(\omega_{A}\right)}{2 \pi \hbar \epsilon_{0} \bar{R}^{3}\left|\epsilon\left(\omega_{A}\right)\right|^{2}}\left\{\left|\frac{\epsilon\left(\omega_{A}\right)+2}{3}\right|^{2}+\frac{\left|\epsilon\left(\omega_{A}\right)\right|^{2}}{9}\right. \\
& \left.-\frac{2}{9} \epsilon_{R}\left(\omega_{A}\right)\left[\epsilon_{R}\left(\omega_{A}\right)+2\right]-\frac{2}{9}\left[\epsilon_{I}\left(\omega_{A}\right)\right]^{2}\right\} .
\end{aligned}
$$

The modifications near a medium resonance are clear. Note that owing to the quantum local-field correction the unspecified parameter $\bar{R}$ also enters into the transverse decay rate. In order to compare our canonical result with that obtained using the classically corrected local field, we use, for comparison, the same Lorentz model for the permittivity of a single-resonance medium as in [3] 4 , 


$$
\epsilon(\omega)=1+\frac{\left(0.46 \omega_{T}\right)^{2}}{\omega_{T}^{2}-\omega^{2}-i \gamma \omega},
$$

where $\omega_{T}$ is the resonance frequency of the medium. Figures 13 show the transverse decay rate $\Gamma^{\perp}$ with and without quantum local-field corrections as a function of the atomic transition frequency $\omega_{A}$ for different values of the damping parameter of the medium, $\gamma$, and the parameter

$$
r=\frac{\lambda_{T}}{\bar{R}} .
$$

First of all, for small $r$, i.e., large virtual-cavity radius $\bar{R}$ one observes little reduction of spontaneous decay for frequencies $\omega_{A}$ just above the resonance frequency $\omega_{T}$. Its possible applications in semiconductor physics and solid-state physics has already been discussed [27].

The greatest difference between the quantum-mechanically and classically corrected transverse decay rates $\Gamma^{\perp}$ and $\Gamma_{\mathrm{cl}}^{\perp}$, respectively, arises near the medium resonance when $\gamma$ is small. Both the imaginary part of the permittivity and the real part can take very large values for $\omega_{A} \approx \omega_{T}$ and in consequence $\Gamma^{\perp}$ can drastically change compared with $\Gamma_{\mathrm{cl}}^{\perp}$. Obviously, in the resonance regime the noise polarization essentially contributes to the local-field and therefore strongly influences $\Gamma^{\perp}$. For small values of $\gamma$ both qualitative and quantitative differences between the rates $\Gamma^{\perp}$ and $\Gamma_{\mathrm{cl}}^{\perp}$ are observed (Fig. 1). With increasing value of $\gamma$ the two rates become less different from each other, the changes being quantitative rather than qualitatitive (compare Fig. 11 with Fig. 3).

Whereas $\Gamma_{\mathrm{cl}}^{\perp}$ is independent of the radius $\bar{R}$ of the virtual cavity, $\Gamma^{\perp}$ sensitively depends on $\bar{R}$. To fix the value of $\bar{R}$ that is undetermined in the Clausius-Mosotti model, experimental data could be used in principle (for recent experiments on spontaneous emission, see, e.g., 15 17). It is worth noting that our theory gives an upper bound $\bar{R}_{\max }$, i.e., a lower bound $r_{\min }$ for the parameter $r$, because of the fact that $\Gamma^{\perp}$ cannot be negative. As already mentioned, the limit $\mathbf{r} \rightarrow \mathbf{r}_{A}$ in Eq. (34) cannot be performed and averaging over a sphere of radius $\bar{R}$ can give negative values, if the radius is not small enough, because the vacuum expectation value of the Hermitian operator $\underline{\hat{\mathbf{E}}}^{\text {loc }}(\mathbf{r}, \omega) \underline{\mathbf{E}}^{\text {loc } \dagger}\left(\mathbf{r}_{A}, \omega\right)$ is not necessarily positive. Figure presents $r_{\min }$ as a function of the damping parameter $\gamma$. The curve was obtained numerically by requiring that $\Gamma^{\perp}$ must not be negative over the whole frequency spectrum.

Figure 1 shows that for chosen (small) $\gamma$ and $r<$ $r_{\text {min }}$ negative values of $\Gamma^{\perp}$ may appear when the atomic transition frequency $\omega_{A}$ approaches the medium resonance frequency $\omega_{T}$ and is in an interval that corresponds to the polariton bandgap between $\omega_{T}$ and $\omega_{L}=$ $\left[\omega_{T}^{2}+\left(0.46 \omega_{T}\right)^{2}\right]^{1 / 2}$ in the Hopfield model of a dielectric in the absence of absorption [23]. Obviously, in this regime of spontaneous decay, in which the decay rate is expected to respond to a change in the local-field noise very sensitively, the Clausius-Mosotti model has to be replaced with a refined model, at least in quantum theory.
From the standard derivation of the (classical) Clausius-Mosotti local-field (see, e.g., [25]) the radius $\bar{R}$ of the virtual cavity should be larger than the average distance of two neighboring atoms but sufficiently smaller than the optical wavelength. In terms of the parameter $r$, the latter requirement means that $r \gg 1$. Provided that the damping parameter $\gamma$ is not too small, this is in agreement with the condition that the parameter $r$ should not be smaller than $r_{\text {min }}$ given in Fig. 1 .

\section{CONCLUSIONS}

Within the frame of the Clausius-Mosotti model we have studied the influence of the quantum local-field correction arising from the noise polarization on the spontaneous decay rate of an excited atom embedded in an absorbing medium. We have shown that inclusion in the local field of the noise polarization ensures that the local field fulfills the fundamental equal-time commutation relations of $\mathrm{QED}$, provided that the static refractive index of the medium does not exceed unity substantially. The calculated rates demonstrate that the contribution of the noise polarization to the local field is extremely important and cannot be ignored. In particular, at the resonance frequencies of the medium the transverse decay rate can drastically change compared with the classically corrected rate where the fluctuating component of the polarization is omitted.

The decay rate crucially depends on the choice of the radius of the virtual cavity. It is worth noting that from the dependence on the radius of the transverse rate - an effect that only results from the noise polarization - a second condition of validity can be imposed on the local-field model. In order to obtain for any transition frequency a positive transverse decay rate, the cavity radius must not exceed some upper bound.

The Clausius-Mosotti virtual-cavity model is commonly based on the assumption that the near field that arises from the atoms inside the cavity averages to zero in general. In quantum optics this assumption may fail, because of the vacuum noise associated with this field, which may be an explanation for the restrictions found. In order to extend the range of validity of the theory, a more refined concept seems to be necessary that considers the near field more carefully. 


\section{APPENDIX A: COMMUTATION RELATIONS OF THE LOCAL FIELD OPERATORS}

From Eq. (23) together with Eq. (22), the local electric field operator reads in Fourier space

$$
\underline{\hat{\mathbf{E}}}^{\mathrm{loc}}(\mathbf{r}, \omega)=\frac{\epsilon(\mathbf{r}, \omega)+2}{3} \hat{\hat{\mathbf{E}}}(\mathbf{r}, \omega)+\frac{i}{3 \epsilon_{0}} \sqrt{\frac{\hbar \epsilon_{0}}{\pi} \epsilon_{I}(\mathbf{r}, \omega)} \hat{\mathbf{f}}(\mathbf{r}, \omega) .
$$

Combining Eqs. (24) and (A1), we obtain for the local magnetic field in Fourier space

$$
\underline{\hat{\mathbf{B}}}^{\mathrm{loc}}\left(\mathbf{r}^{\prime}, \omega^{\prime}\right)=\nabla \times\left[\mathcal{P} \frac{1}{i \omega^{\prime}} \hat{\mathbf{E}}\left(\mathbf{r}^{\prime}, \omega^{\prime}\right) \frac{\epsilon\left(\mathbf{r}^{\prime}, \omega^{\prime}\right)+2}{3}+\frac{1}{3 \epsilon_{0}} \mathcal{P} \frac{1}{\omega^{\prime}} \sqrt{\frac{\hbar \epsilon_{0}}{\pi} \epsilon_{I}\left(\mathbf{r}^{\prime}, \omega^{\prime}\right)} \hat{\mathbf{f}}\left(\mathbf{r}^{\prime}, \omega^{\prime}\right)\right] .
$$

Recalling Eqs. (25) and (26), from Eqs. (A1) and (A2) together with Eq. (16) the local electric and magnetic fields are given by

$$
\begin{aligned}
\hat{E}_{i}^{\text {loc }}(\mathbf{r})= & \sqrt{\frac{\hbar \epsilon_{0}}{\pi}} \int_{0}^{\infty} d \omega \int d^{3} \mathbf{s}\left[\frac{\epsilon(\mathbf{r}, \omega)+2}{3 \epsilon_{0}} \frac{i \omega^{2}}{c^{2}} \sqrt{\epsilon_{I}(\mathbf{s}, \omega)} G_{i j}(\mathbf{r}, \mathbf{s}, \omega) \hat{f}_{j}(\mathbf{s}, \omega)+\text { H.c. }\right] \\
& +\frac{1}{3 \epsilon_{0}} \sqrt{\frac{\hbar \epsilon_{0}}{\pi}} \int_{0}^{\infty} d \omega\left[i \sqrt{\epsilon_{I}(\mathbf{r}, \omega)} \hat{f}_{i}(\mathbf{r}, \omega)+\text { H.c. }\right], \\
\hat{B}_{k}^{\text {loc }}\left(\mathbf{r}^{\prime}\right)= & \epsilon_{k l m} \partial_{l}^{r^{\prime}}\left\{\sqrt{\frac{\hbar \epsilon_{0}}{\pi}} \mathcal{P} \int_{0}^{\infty} d \omega^{\prime} \int d^{3} \mathbf{s}^{\prime}\left[\frac{\epsilon\left(\mathbf{r}^{\prime}, \omega^{\prime}\right)+2}{3 \epsilon_{0}} \frac{\omega^{\prime}}{c^{2}} \sqrt{\epsilon_{I}\left(\mathbf{s}^{\prime}, \omega^{\prime}\right)} G_{m n}\left(\mathbf{r}^{\prime}, \mathbf{s}^{\prime}, \omega^{\prime}\right) \hat{f}_{n}\left(\mathbf{s}^{\prime}, \omega^{\prime}\right)+\text { H.c. }\right]\right. \\
& \left.+\frac{1}{3 \epsilon_{0}} \sqrt{\frac{\hbar \epsilon_{0}}{\pi}} \mathcal{P} \int_{0}^{\infty} \frac{d \omega^{\prime}}{\omega^{\prime}}\left[\sqrt{\epsilon_{I}\left(\mathbf{r}^{\prime}, \omega^{\prime}\right)} \hat{f}_{m}\left(\mathbf{r}^{\prime}, \omega^{\prime}\right)+\text { H.c. }\right]\right\} .
\end{aligned}
$$

Thus, the (equal-time) commutator between the local electric and magnetic fields can be given by

$$
\begin{aligned}
& {\left[\hat{E}_{i}^{\mathrm{loc}}(\mathbf{r}), \hat{B}_{k}^{\mathrm{loc}}\left(\mathbf{r}^{\prime}\right)\right]} \\
& =\frac{\hbar \epsilon_{0}}{\pi} \epsilon_{k l m} \partial_{l}^{r^{\prime}}\left\{\mathcal{P} \int_{0}^{\infty} d \omega \int d^{3} \mathbf{s}\left[\frac{i \omega^{3}}{c^{4}} \frac{\epsilon(\mathbf{r}, \omega)+2}{3 \epsilon_{0}} \frac{\epsilon^{*}\left(\mathbf{r}^{\prime}, \omega\right)+2}{3 \epsilon_{0}} \epsilon_{I}(\mathbf{s}, \omega) G_{i j}(\mathbf{r}, \mathbf{s}, \omega) G_{m j}^{*}\left(\mathbf{r}^{\prime}, \mathbf{s}, \omega\right)-\text { c.c. }\right]\right. \\
& +\frac{1}{3 \epsilon_{0}} \mathcal{P} \int_{0}^{\infty} d \omega\left[\frac{\epsilon^{*}\left(\mathbf{r}^{\prime}, \omega\right)+2}{3 \epsilon_{0}} \frac{i \omega}{c^{2}} \epsilon_{I}(\mathbf{r}, \omega) G_{m i}^{*}\left(\mathbf{r}^{\prime}, \mathbf{r}, \omega\right)-\text { c.c. }\right] \\
& +\frac{1}{3 \epsilon_{0}} \mathcal{P} \int_{0}^{\infty} d \omega\left[\frac{\epsilon(\mathbf{r}, \omega)+2}{3 \epsilon_{0}} \frac{i \omega}{c^{2}} \epsilon_{I}\left(\mathbf{r}^{\prime}, \omega\right) G_{i m}\left(\mathbf{r}, \mathbf{r}^{\prime}, \omega\right)-\text { c.c. }\right] \\
& \left.+\frac{2 i}{9 \epsilon_{0}^{2}} \mathcal{P} \int_{0}^{\infty} \frac{d \omega}{\omega}\left[\epsilon_{I}(\mathbf{r}, \omega) \delta_{i m} \delta\left(\mathbf{r}-\mathbf{r}^{\prime}\right)\right]\right\} .
\end{aligned}
$$

The remaining spatial integral in Eq. (A5) can be calculated using the symmetry relation

$$
G_{i j}\left(\mathbf{r}, \mathbf{r}^{\prime}, \omega\right)=G_{j i}\left(\mathbf{r}^{\prime}, \mathbf{r}, \omega\right),
$$

the crossing relation

$$
G_{i j}\left(\mathbf{r}, \mathbf{r}^{\prime}, \omega\right)=G_{i j}^{*}\left(\mathbf{r}, \mathbf{r}^{\prime},-\omega\right)
$$

and the integral relation 20]

$$
\frac{\omega^{2}}{c^{2}} \int d^{3} \mathbf{s} \epsilon_{I}(\mathbf{s}, \omega) G_{l i}(\mathbf{s}, \mathbf{r}, \omega) G_{l j}^{*}\left(\mathbf{s}, \mathbf{r}^{\prime}, \omega\right)=\frac{1}{2 i}\left[G_{j i}\left(\mathbf{r}^{\prime}, \mathbf{r}, \omega\right)-G_{i j}^{*}\left(\mathbf{r}, \mathbf{r}^{\prime}, \omega\right)\right] .
$$

Straightforward calculation yields

$$
\left[\hat{E}_{i}^{\mathrm{loc}}(\mathbf{r}), \hat{B}_{k}^{\mathrm{loc}}\left(\mathbf{r}^{\prime}\right)\right]=\frac{\hbar}{\pi \epsilon_{0}} \epsilon_{k l m} \partial_{l}^{r^{\prime}}\left[I_{i m}^{(1)}\left(\mathbf{r}, \mathbf{r}^{\prime}\right)+I_{i m}^{(2)}\left(\mathbf{r}, \mathbf{r}^{\prime}\right)\right],
$$


where

$$
\begin{gathered}
I_{i m}^{(1)}\left(\mathbf{r}, \mathbf{r}^{\prime}\right)=\mathcal{P} \int_{-\infty}^{\infty} d \omega \frac{\omega}{c^{2}} G_{i m}\left(\mathbf{r}, \mathbf{r}^{\prime}, \omega\right)\left\{1+\frac{1}{3}[\epsilon(\mathbf{r}, \omega)-1]+\frac{1}{3}\left[\epsilon\left(\mathbf{r}^{\prime}, \omega\right)-1\right]+\frac{1}{9}[\epsilon(\mathbf{r}, \omega)-1]\left[\epsilon\left(\mathbf{r}^{\prime}, \omega\right)-1\right]\right\}, \\
I_{i m}^{(2)}\left(\mathbf{r}, \mathbf{r}^{\prime}\right)=\mathcal{P} \int_{-\infty}^{\infty} d \omega \frac{\epsilon_{I}(\mathbf{r}, \omega)}{\omega} \delta_{i m} \delta\left(\mathbf{r}-\mathbf{r}^{\prime}\right) .
\end{gathered}
$$

Closing the integration contour in the upper complex frequency half-plane and following the line in [18], we derive that

$$
I_{i m}^{(1)}\left(\mathbf{r}, \mathbf{r}^{\prime}\right)=i \pi \delta_{i m} \delta\left(\mathbf{r}-\mathbf{r}^{\prime}\right) .
$$

Recalling the Kramers-Kronig relations, the $\omega$ integral in Eq. (A11) is easily performed to obtain

$$
I_{i m}^{(2)}\left(\mathbf{r}, \mathbf{r}^{\prime}\right)=\pi\left[\epsilon_{R}(\mathbf{r}, 0)-1\right] \delta_{i m} \delta\left(\mathbf{r}-\mathbf{r}^{\prime}\right)=\pi[\epsilon(\mathbf{r}, 0)-1] \delta_{i m} \delta\left(\mathbf{r}-\mathbf{r}^{\prime}\right) .
$$

Combining Eqs. (A9), (A12), and (A13) then yields the commutation relation (27). 
[1] P.W. Milonni, The Quantum Vacuum: An Introduction to Quantum Electrodynamics, (Academic, San Diego, 1994).

[2] R.J. Glauber and M. Lewenstein, Phys. Rev. A 43, 467 (1991).

[3] S.M. Barnett, B. Huttner, and R. Loudon, Phys. Rev. Lett. 68, 3698 (1992).

[4] S.M. Barnett, B. Huttner, R. Loudon, and R. Matloob, J. Phys. B: At. Mol. Opt. Phys. 29, 3763 (1996).

[5] G. Juzeliūnas, Phys. Rev A 55, R4015 (1997).

[6] A. Tip, Phys. Rev. A 56, 5022 (1997).

[7] J. Knoester and S. Mukamel, Phys. Rev. A 40, 7065 (1989).

[8] R.G. Hulet, E.S. Hilfer, and D. Kleppner, Phys. Rev. Lett. 55, 2137 (1985).

[9] W. Jhe, A. Anderson, E.A. Hinds, D. Meschede, L. Moi, and S. Haroche, Phys. Rev. Lett. 58, 1320 (1987).

[10] K. Koshino and A. Shimizu, Phys. Rev. A 53, 4468 (1996).

[11] I. Takahashi and K. Ujihara, Phys. Rev. A 56, 2299 (1997).

[12] M.S. Tomaš and Z. Lenac, Phys. Rev. A 56, 4197 (1997).

[13] M.S. Yeung and T.K. Gustafson, Phys. Rev. A 54, 5227 (1996).

[14] W. Barnes, J. Mod. Opt. 45, 661 (1998) and references cited therein.

[15] G.L.J.A. Rikken and Y.A.R.R. Kessener, Phys. Rev. Lett. 74, 880 (1995).

[16] F.J.P. Schuurmans, D.T.N. de Lang, G.H. Wegdam, R. Sprik, and A. Lagendijk, Phys. Rev. Lett. 80, 5077 (1998).

[17] P. de Vries and A. Lagendijk, Phys. Rev. Lett. 81, 1381 (1998).

[18] S. Scheel, L. Knöll, and D.-G. Welsch, Phys. Rev A 58, 700 (1998).

[19] T. Gruner and D.-G. Welsch, Phys. Rev. A 53, 1818 (1996).

[20] Ho Trung Dung, L. Knöll, and D.-G. Welsch, Phys. Rev. A 57, 3931 (1998).

[21] R. Matloob, R. Loudon, S.M. Barnett, and J. Jeffers, Phys. Rev. A 52, 4823 (1995). R. Matloob and R. Loudon, Phys. Rev. A 53, 4567 (1996).

[22] B. Huttner and S.M. Barnett, Phys. Rev. A 46, 4306 (1992).

[23] J.J. Hopfield, Phys. Rev. 112, 1555 (1958).

[24] U. Fano, Phys. Rev. A 103, 1202 (1956).

[25] J.D. Jackson, Classical Electrodynamics, (Wiley, New York, 1975).

[26] C.M. Bowden and J.P. Dowling, Phys. Rev. A 47, 1247 (1993).

[27] E. Yablonovitch, Phys. Rev Lett. 58, 2059 (1987).

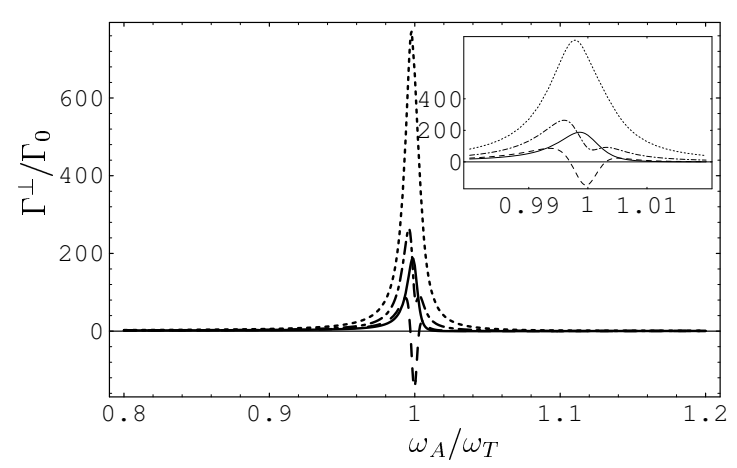

FIG. 1. The transverse decay rate $\Gamma^{\perp}$ is shown as a function of the transition frequency $\omega_{A}$ for $\gamma=0.01$ and $r=10$ (dashed curve), $r=20$ (broken curve) and $r=30$ (dotted curve). For comparison, the rate without quantum local-field correction [3] is shown (solid curve). Since for $r=10$ (broken curve) $\Gamma^{\perp}$ becomes negative, this case must be excluded from consideration (cf. Fig. 4 ).

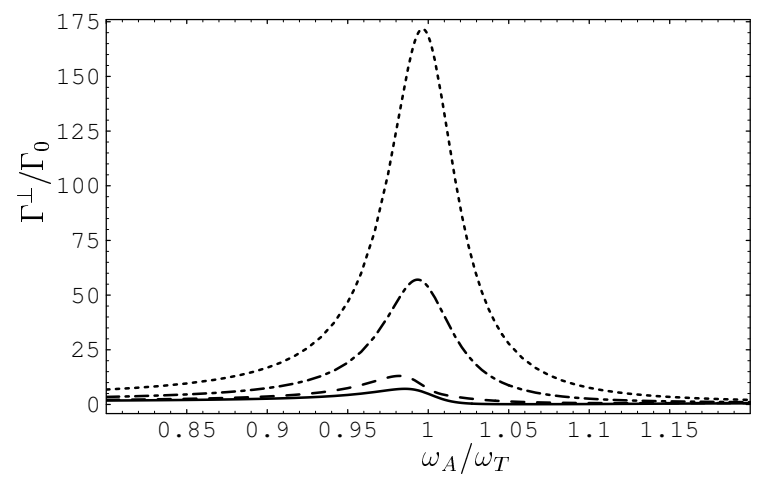

FIG. 2. The transverse decay rate $\Gamma^{\perp}$ is shown as a function of the transition frequency $\omega_{A}$ for $\gamma=0.05$ and $r=10$ (dashed curve), $r=20$ (broken curve), and $r=30$ (dotted curve). For comparison, the rate without quantum local-field correction [3] is shown (solid curve).

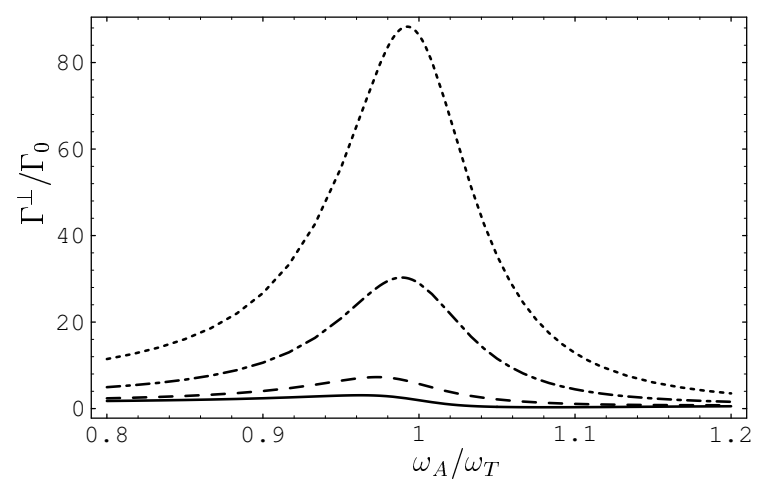


FIG. 3. The transverse decay rate $\Gamma^{\perp}$ is shown as a function of the transition frequency $\omega_{A}$ for $\gamma=0.1$ and $r=10$ (dashed curve), $r=20$ (broken curve), and $r=30$ (dotted curve). For comparison, the rate without quantum local-field correction [3] is shown (solid curve).

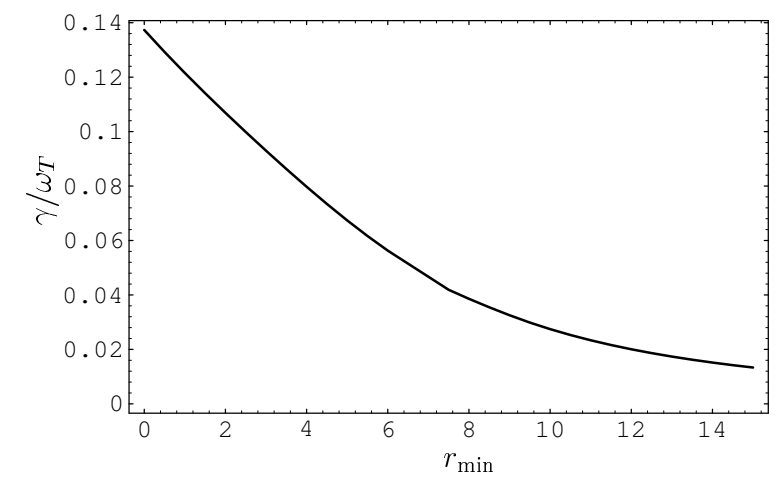

FIG. 4. The lower bound $r_{\text {min }}$ of the parameter $r$, Eq. (52), is shown as a function of the damping parameter $\gamma$. The region below the curve is the part where the transverse decay rate $\Gamma^{\perp}$ may take negative values and is therefore forbidden. 